\title{
Cardiovascular Risk in Rheumatoid Arthritis (RA): Does It Matter If RA Is Diagnosed in Early or Late Age?
}

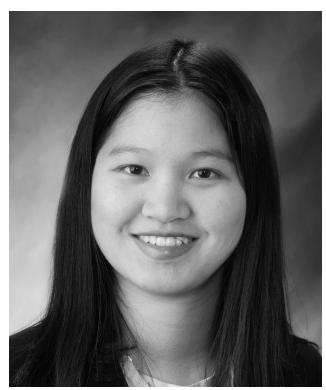

Rheumatoid arthritis (RA) is a complex autoimmune disorder with heterogeneous clinical presentations, including age at disease onset. Indeed, RA can be diagnosed at any age. Although the peak age of onset is between 50 and 75 years, the prevalence of RA in females over age 65 is up to $5 \%{ }^{1}$. Given the prevalence of RA is about $1 \%$ in whites ${ }^{2}$, there is a substantial subgroup of RA patients who are elderly (over age 65) at disease onset. There are over 50 countries with current life expectancies of 75 years or older $^{3}$. Hence, the risk of morbidity and mortality even in the subgroup of elderly-onset RA patients is of clinical relevance.

The approximately 2 to 3 -fold increased risk of cardiovascular disease (CVD) in RA is well-established ${ }^{4,5,6}$. It is suggested that both traditional $\mathrm{CV}$ risk factors as well as RA-specific disease mechanisms (e.g., systemic inflammation, immune dysregulation, and premature immunosenescence) contribute to the overall $\mathrm{CV}$ risk in RA. However, few studies have examined the risks and determinants of CVD in subgroups of RA patients depending on their age at diagnosis. In a previous population-based study $^{6}$, it was shown that the 10 -year absolute cardiovascular $(\mathrm{CV})$ risk in given age groups varied substantially according to the presence of $\mathrm{CV}$ risk factors; among 60 to 69-year-old RA patients with no risk factors, the absolute CV risk was only $16.8 \%$, but rose to $60.4 \%$ if CV risk factors of smoking, hypertension (HTN), dyslipidemia, diabetes, and obesity were present. A recent study by Ajeganova and colleagues in this issue of The Journal ${ }^{7}$ complements these findings by examining the effect of early RA disease factors on CV risk, stratified by age at RA disease onset.

Their study sought to examine the associations of RA disease-related factors within the first 2 years of diagnosis and incident CVD morbidity and all-cause mortality, specifically comparing these effects between subgroups of RA patients diagnosed at early $(<65 \mathrm{yrs})$ versus late $(\geq 65 \mathrm{yrs})$ age. Their study was well-suited to address this because they used data from an established early RA Swedish cohort called BARFOT, which is a multicenter prospective observational study including both urban and rural referral areas. Hence, they could identify incident RA cases (with disease duration $\leq 12$ months), and exclude cases with prevalent CVD at RA diagnosis and those $<20$ years old. The study subjects were recruited from 1993 to 1999. The study design allowed for a robust followup time until 2010.

The investigators found that 177 CVD events and 151 deaths (of which 50\% were CVD-related) occurred during the median 13-year observation period in the study population of 741 RA subjects. In early-onset RA patients, the incident CVD event rate was 1.0 per 100 person-years, and all-cause mortality was 0.8 per 100 person-years; while in patients with late-onset RA, the incident CVD event rate was 5.9 per 100 person-years and all-cause mortality was 4.2 per 100 person-years. This is consistent with previous observations ${ }^{6}$ that confirmed that age is the key determinant of CVD in both RA and non-RA subjects.

Seropositivity for rheumatoid factor (RF), anticitrullinated peptide antibodies (ACPA), white blood (cell) count (WBC) at diagnosis, area under the curve (AUC) of C-reactive protein (CRP), erythrocyte sedimentation rate (ESR), and visual analog scale (VAS) pain were associated with higher CVD risk among patients with early-onset RA. In late-onset RA, improved CVD prognosis was associated with reductions in CRP, ESR, and health assessment questionnaire (HAQ) during the first year, as well as regular use of methotrexate (MTX), whereas regular use of glucocorticoids (GC) worsened CVD outcome. This corroborates hypotheses of the significance of inflammation on $\mathrm{CV}$ risk in RA, as well as known adverse metabolic effects of GC that impart additional traditional $\mathrm{CV}$ risks. Of note, although higher WBC values at baseline were found to be associated with worse CVD outcomes in younger RA patients, the absolute differences in WBC values were of questionable clinical significance and were not in leukocytosis ranges ( 8.7 vs 8.1 for presence vs absence of incident CVD events; 8.9 vs 8.0 for death vs survival). However, the frequency of regular use of GC during obser-

See Disease factors in early RA and age-related CVD risk, page 1958

Personal non-commercial use only. The Journal of Rheumatology Copyright () 2013. All rights reserved. 
vation, WBC, RF positivity, VAS pain, and HAQ scores at baseline did not differ between the age groups, so the association of a higher WBC with CVD outcomes in the younger group cannot be attributed simply to higher GC use or higher initial disease activity in that group; the WBC was actually measured prior to commencement of GC. In fact, the older group had higher baseline CRP, ESR, and disease activity score-28 (DAS28). Interestingly, in the general population, higher WBC has also been found to be independently associated with CVD and mortality ${ }^{8,9}$. Although the magnitude of these associations of WBC with $\mathrm{CV}$ risk is small, further investigations in RA populations are warranted.

Regarding all-cause mortality, similar findings were seen: RF or ACPA seropositivity, higher WBC at baseline, and AUC of VAS pain over the first 2 years were associated with increased mortality only in the younger group. Among the older group, AUC of DAS28 and regular use of GC increased mortality risk. Notably, although regular GC use was not statistically significantly associated with incident CVD or mortality in the younger group, there was still a trend in this direction (see Figure 1 of Ajeganova, et $a l)^{7}$. In both younger and older groups, higher AUC of CRP, ESR, and HAQ, as well as HAQ score after 2 years, were independently and significantly associated with poorer survival. This finding again corroborates hypotheses that there is likely a "window of opportunity" in which tight control of inflammation shortly after disease onset attenuates $\mathrm{CV}$ risk in RA. The results suggest that such reductions in CRP, ESR, and DAS28 within 1 year in patients with elderly onset RA may be particularly protective. They also suggest a deleterious, rather than beneficial, effect of regular GC use in elderly RA patients. The effect of GC on $\mathrm{CV}$ risk in RA is still unclear, as this effect is particularly difficult to ascertain in cohort studies due to confounding by indication. There are also opposing theories, with both beneficial (antiinflammatory) and harmful (metabolic dysregulation) effects of $\mathrm{GC}$ on CVD risk being recognized. There has also been evidence that GC use may increase CV risk in only seropositive, but not seronegative RA patients ${ }^{10}$, suggesting different mechanisms of GC-driven CVD, depending on the presence of RF. Given other comorbidities that may exist (e.g., osteoporosis or peptic ulcer disease), even more caution is prudent when considering using GC in elderly patients with RA. It should be noted that the authors of this study did not have information on additional comorbidities that may have confounded their analyses. Additionally, they did not have information on extraarticular manifestations of RA, e.g., nodules and vasculitis, which have been shown previously to independently predict higher $\mathrm{CV}$ risk $^{11}$. Since these features were not included in the multivariate models, one cannot rule out differential effects due to such factors.

In considering the conclusions from Ajeganova and colleagues' study, one must bear in mind the limitation of confounding by indication in terms of treatment in younger versus older patients with RA. Specifically, elderly-onset RA patients will likely be treated differently in terms of disease-modifying antirheumatic drug (DMARD) use, due to comorbidities that exist in the elderly. The authors note that the frequency of use of DMARD, MTX, and GC during the first year, and regular use of GC during observation did not differ between the younger versus older groups. They suggest that at least initially in the RA disease course, any comorbidity difference between the groups did not affect therapy choice. Only later in the observation period was the older RA group treated less often with MTX and biologics regularly; this is in accordance with recommendations in Sweden during the study period. In the United States and other countries, there may be more heterogeneity in how rheumatologists choose therapies for patients with RA depending on their age at diagnosis. Further, with wider recognition of the importance of early tight disease control in RA after the recruitment period of this study, practice patterns have likely changed for the use of therapies during the first year of disease in both younger and older RA patients. Future studies are needed to examine the differential modern treatment effects on $\mathrm{CV}$ risk in both younger and older RA subgroups.

Despite these limitations, inherent in retrospective studies, the contribution by Ajeganova and colleagues ${ }^{7}$ is important, as it reminds the rheumatologist of the importance of early tight disease control even in their elderly-onset RA patients. This subgroup is often treated differently, with DMARD/biologic therapies sometimes withheld simply due to age, despite lower remission rates, suggesting a potential treatment deficit in older patients ${ }^{12}$. With regard to $\mathrm{CV}$ risk in RA, given that age is already their major risk factor for an incident CVD event, we should perhaps pay even closer attention to modifying other risk factors. Further, GC use should be minimized as much as possible in patients with elderly-onset RA, not only because of higher risk of toxicities from comorbidities, but also because of its association with CV morbidity and mortality in this age group.

\section{KIMBERLY P. LIANG, MD,}

\author{
Department of Medicine and Division of Rheumatology \\ and Clinical Immunology, \\ University of Pittsburgh, \\ Pittsburgh, Pennsylvania, USA.
}

Address correspondence to Dr. Liang, University of Pittsburgh, Division of Rheumatology, 3500 Terrace St., BST S723, Pittsburgh, PA 15261, USA.E-mail: liangkp@upmc.edu

\section{REFERENCES}

1. Schur PH, Gabriel SE, Crowson CS. Epidemiology of risk factors for and possible causes of rheumatoid arthritis. [Internet. Accessed October 2, 2013.] Available from: http://www.uptodate.com/contents/

Personal non-commercial use only. The Journal of Rheumatology Copyright @ ${ }^{2013}$. All rights reserved. 
epidemiology-of-risk-factors-for-and-possible-causes-ofrheumatoid-arthritis?

2. Spector TD. Rheumatoid arthritis. Rheum Dis Clin North Am 1990;16:513-37.

3. List of countries by life expectancy. [Internet. Accessed October 2, 2013.] Available from: http://en.wikipedia.org/wiki/ List_of_countries_by_life_expectancy

4. Solomon DH, Karlson EW, Rimm EB, Cannuscio CC, Mandl LA, Manson JE, et al. Cardiovascular morbidity and mortality in women diagnosed with rheumatoid arthritis. Circulation 2003;107:1303-7.

5. Maradit-Kremers H, Nicola PJ, Crowson CS, Ballman KV, Gabriel SE. Cardiovascular death in rheumatoid arthritis: a population-based study. Arthritis Rheum 2005;52:722-32.

6. Kremers HM, Crowson CS, Therneau TM, Roger VL, Gabriel SE. High ten-year risk of cardiovascular disease in newly diagnosed rheumatoid arthritis patients: a population-based cohort study. Arthritis Rheum 2008;58:2268-74.

7. Ajeganova S, Andersson ML, Frostegård J, Hafström I. Disease factors in early rheumatoid arthritis are associated with differential risks for cardiovascular events and mortality depending on age at onset: A 10-year observational cohort study. J Rheumatol 2013;40:1958-66.

8. Taglieri N, Bacchi Reggiani ML, Palmerini T, Cinti L, Saia F, Guastaroba P, et al. Baseline white blood cell count is an independent predictor of long-term cardiovascular mortality in patients with non-ST-segment elevation acute coronary syndrome, but it does not improve the risk classification of the GRACE score. Cardiology 2013;124:97-104.

9. Palmerini T, Généreux P, Mehran R, Dangas G, Caixeta A, Riva $\mathrm{DD}$, et al. Association among leukocyte count, mortality, and bleeding in patients with non-ST-segment elevation acute coronary syndromes (from the Acute Catheterization and Urgent Intervention Triage StrategY [ACUITY] trial). Am J Cardiol 2013;111:1237-45.

10. Davis JM 3rd, Maradit Kremers H, Crowson CS, Nicola PJ, Ballman KV, Therneau TM, Roger VL, Gabriel SE.

Glucocorticoids and cardiovascular events in rheumatoid arthritis: a population-based cohort study. Arthritis Rheum 2007;56:820-30.

11. Turesson C, McClelland RL, Christianson TJ, Matteson EL. Severe extra-articular disease manifestations are associated with an increased risk of first ever cardiovascular events in patients with rheumatoid arthritis. Ann Rheum Dis 2007;66:70-5.

12. Huscher D, Sengler C, Gromnica-Ihle E, Bischoff S, Eidner T, Ochs $\mathrm{W}$, et al. Clinical presentation, burden of disease and treatment in young-onset and late-onset rheumatoid arthritis: a matched-pairs analysis taking age and disease duration into account. Clin Exp Rheumatol 2013;31:256-62.

J Rheumatol 2013;40:1945-7; doi:10.3899/jrheum.131109 